\title{
Caractérisation des insectes et des champignons infestant les fèves de cacao dans les principales zones de production en Côte d'Ivoire
}

\author{
Brou Julien Kouiakou \\ Programme de Recherche sur le Coton, \\ Centre National de Recherche Agronomique (CNRA), Côte d'Ivoire \\ Bi Zahouli Irie \\ Laboratoire de Chimie-Technologie, \\ Centre National de Recherche Agronomique (CNRA), Côte d'Ivoire \\ Kouadio Emmanuel N'goran \\ Programme de Recherche sur le Coton, \\ Centre National de Recherche Agronomique (CNRA), Côte d'Ivoire \\ Brou Kouame \\ Programme de Gestion Durable des Sols et Maîtrise de l'Eau, \\ Centre National de Recherche Agronomique (CNRA), Côte d'Ivoire \\ Acka Emmanuel Dick \\ Daouda Kone \\ Laboratoire de Physiologie Végétale, \\ Université Félix Houphouët-Boigny, Côte d'Ivoire
}

Doi: 10.19044/esj.2018.v14n33p298 URL:http://dx.doi.org/10.19044/esj.2018.v14n33p298

\begin{abstract}
If the culture of the cocoa in Côte d'Ivoire gives a great satisfaction concerning the production level, the quality of raw cocoa beans remains to be improved. One of the causes of this depreciation is the moulds development in beans. In order to identify responsible fungus, cocoa beens have been collected in the maine production areas which are the East, the Mid-West and the South-West, represented respectively by the localities of Abengourou, Oumé and Soubré. From collected beans, the fungus have been insulated and purified on PDA medium and then, they have been identified by observing their morphocultural and microscopic characteristics. The results show that 9 mushroom species belonging to 5 genera were identified in cocoa beans collected in the whole of the localities. There are Aspergillus, Penicillium, Fusarium, Curvularia and Streptomyces. Among them, the Aspergillus genus which is able to produce ochratoxine, was found with an appearance frequency of $86.30 \%$.
\end{abstract}


Keywords: Beans, raw cocoa, insect, fungus, quality, Côte d'Ivoire

\section{Résumé}

Si la culture du cacao en Côte d'Ivoire donne une grande satisfaction au niveau de la production, la qualité des fèves reste à améliorer. Une des causes de cette dépréciation est l'infestation des fèves par les moisissures et les insectes. En vue de les identifier, des fèves de cacao marchand ont été collectées dans les pricipales zones de production que sont 1'Est, le CentreOuest et le Sud-Ouest, représentées respectivement par les localités d'Abengourou, Oumé et Soubré. Les insectes retrouvés dans les fèves on été identifiés par observation de leur caractéristiques morphologiques à l'œil nu sous une lumière blanche ou et à la loupe. A partir des fèves collectées, les champignons ont été isolés et purifiés sur milieu PDA (Potato Dextrose Agar) puis leurs genres ont été identifiées en s'appuyant sur leurs caractères morphoculturaux et microscopiques. Les résultats montrent qu'Ephestia cautella est l'insecte qui déprécie les fèves. Il se retrouve sous forme de larve de papillon. Par ailleurs, 9 espèces de champignons appartenant à 5 genres différents ont été identifiées dans les fèves de cacao collectées dans l'ensemble des localités. Ce sont les genres Aspergillus, Penicillium, Fusarium, Curvularia et Streptomyces. Parmi eux, le genre Aspergillus susceptible de produire de l'ochratoxine, a été retrouvé avec une fréquence d'apparition de $86.30 \%$.

Mots clés: Fèves, cacao marchand, insecte, champignon, qualité, Côte d'Ivoire

\section{Introduction}

L'économie de la Côte d'Ivoire, à l'instar de celle de la plupart des pays en voie de développement, est tributaire de l'exportation de matières premières agricoles au nombre desquelles figure le cacao (Jarrige et Ruf, 2000). Premier produit d'exportation, le cacao est produit dans la plupart dans les zones forestières du pays dont l'Est, le Centre-Ouest et le Sud-Ouest en sont les principales (Kouakou et al. 2013). La bonne politique agricole a considérablement favorisé l'essor de la cacaoculture en Côte d'Ivoire, de sorte que depuis 1970, le pays est le leader mondial du cacao avec en moyenne 1,2 millions de tonnes produites par an, soit près de $40 \%$ de la production mondiale (ICCO, 2005). En dépit de cette performance, il existe des problèmes de qualité au niveau des fèves. Cette dépréciation est souvent liée aux mauvaises pratiques au champ et à la non maîtrise des activités de récolte et poste récolte (Cros et Jeanjean, 1995; Aneani et Takrama, 2006, Bastide et al., 2010), mais aussi aux infestations par les champignons et les insectes de stock 
(Lery, 1974) . La plupart du temps, ces infestations sont constatées après achat du cacao, c'est-à-dire durant la phase de commercialisation ou de stockage des fèves avant leur transformation. Alors que les insectes se nourrissent des fèves à l'intérieur desquelles ils se logent, les champignons quant à eux, se développent sous forme de moisissures. Leur taux élevé donne non seulement un mauvais grade commercial au cacao, mais aussi, certains d'entre eux sont susceptibles de produire des substances toxiques telles que l'ochratoxine et l'aflatoxine fortement redoutées par les consommateurs (FAO, 1999). La présente étude a été effectuée sur des fèves marchands, collectées dans les principales zones de production du cacao en Côte d'Ivoire. Elle avait pour objectif de connaitre la nature des insectes et des champignons infestant les fèves de cacao en vue d'appréhender les risques et proposer éventuellement des moyens pour éviter leur prolifération.

\section{Matériel et méthodes}

\section{Prélèvement et conservation des échantillons de fèves destinées aux analyses:}

Trente échantillons de $3000 \mathrm{~g}$ de fève de cacao marchand chacun ont été prélevés dans les principales zones de production du cacao que sont l'Est, le Centre-Ouest et le Sud-Ouest, respectivement représentés par les localités d'Abengourou, Oumé et Soubré.

Pour l'identification des insectes, des lots de $1500 \mathrm{~g}$ de fèves de cacao chacun ont été d'abord conditionnés dans des sacs en tissu de coton (figure 1a). Puis, ceux-ci ont été placés à l'intérieur d'un autre sac fait en treillis de mousseline (figure 1b). Enfin, l'ensemble a été conservé pendant trois mois dans une cage recouverte de moustiquaire de dimension $2 \mathrm{~m} \times 1,8 \mathrm{~m} \times 1,1 \mathrm{~m}$ (figure 2). Dans le système, chaque échantillon est isolé des autres, et l'ensemble des échantillons est à l'abri des insectes ravageurs. La température et l'hygrométrie journalières de l'enceinte de stockage ont respectivement variées entre 26 et $34{ }^{\circ} \mathrm{C}$ et entre 53 et $90 \%$.

Pour l'identification des champignons, une masse de $500 \mathrm{~g}$ de fèves de cacao prélevées dans les échantillons collectés dans les localités de production, a été conditionnée dans des sachets plastiques hermétiquement fermés en vue d'éviter tout contact avec l'extérieur. 

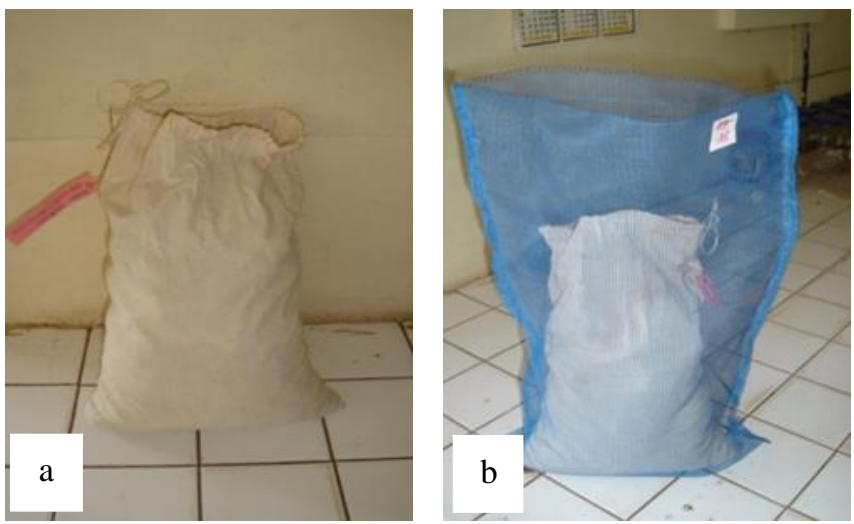

Figure 1 : Echantillons de fèves de cacao durant leur conservation :

a : sac en coton contenant les fèves de cacao (Echelle : 1/10)

$\mathrm{b}$ : sac en coton contenant les fèves de cacao et placé dans un autre en mousseline (Echelle : $1 / 13)$

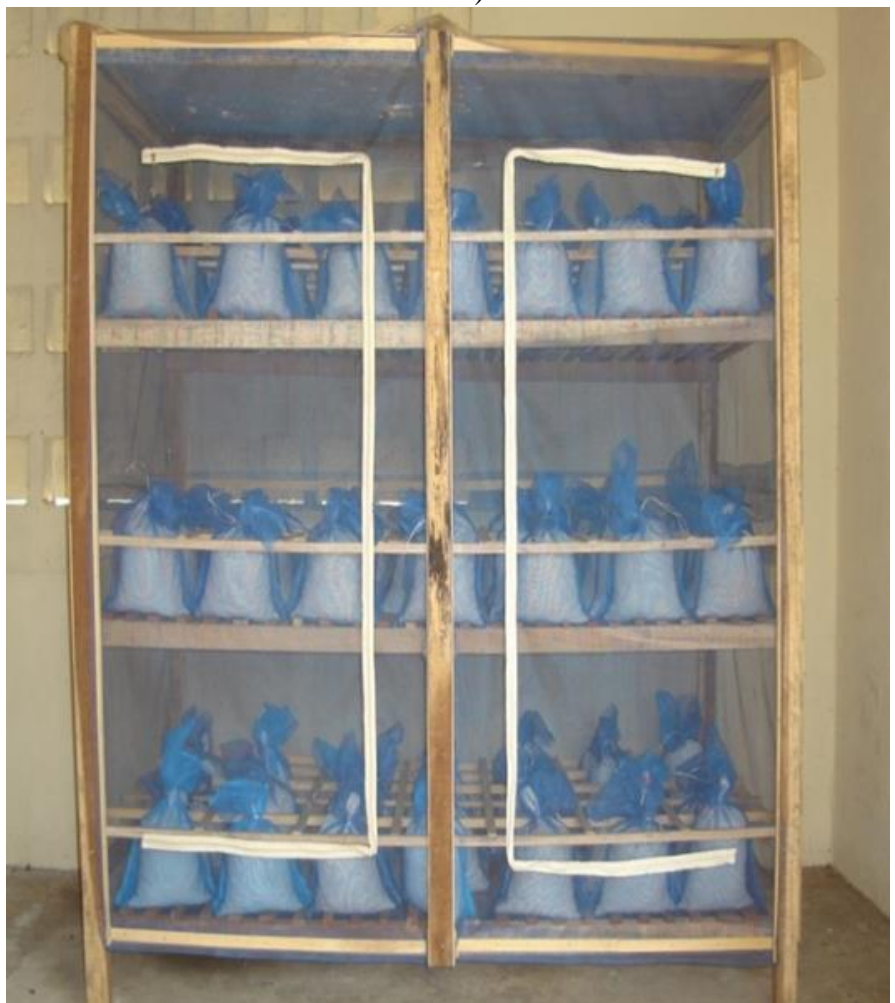

Figure 2 : Echantillons de fèves de cacao durant leur conservation (Echelle : 1/20)

Observation et identification des insectes et espèces fongiques infestant les fèves

Observation et identification des insectes

L'identification des insectes retrouvés dans les fèves a été faite sur les échantillons entreposés pendant trois mois, par observation à l'œil nu sous une 
lumière blanche. Elle s'est effectuée grâce à la classification proposée par Stanek (1979). Celle-ci s'appuie sur la subdivision du corps, le nombre d'antennes et les caractéristiques des ailles (nombre, taille et forme) pour identifier les insectes retrouvés dans les échantillons.

\section{Observation et identification des champignons}

Les champignons infestant les fèves sous forme de moisissures ont été identifiés par la méthode d'étalement, d'isolement et de purification sur milieu de culture PDA (Potato Dextrose Agar) (Kholkhal, 2006 ; Mounjouenpou et al., 2009).

\section{Préparation des extraits fongiques:}

A partir de $100 \mathrm{~g}$ de chaque échantillon, un échantillon composite a été fait dans chaque localité. Ainsi, 3 échantillons représentant les 3 zones d'étude ont été obtenus. Une masse de $100 \mathrm{~g}$ de chaque échantillon composite a ensuite été broyée à l'aide d'un broyeur à hélice de type Moulinex. L'extrait fongique a été préparé par homogénéisation dans $90 \mathrm{ml}$ d'eau distillée stérile, de $10 \mathrm{~g}$ de broyat de l'échantillon composite. L'isolement a été réalisé sur des concentrations allant de $10^{-1}$ à $10^{-7} \mathrm{~g} / \mathrm{ml}$ par dilutions successives de l'homogénat.

\section{Isolement, identification et calcul des fréquences de contamination}

L'isolement a été effectué sur des boîtes de Pétri de $90 \mathrm{~mm}$ de diamètre contenant un milieu PDA, auquel $5 \mathrm{ml}$ d'auréomycine et $5 \mathrm{ml}$ de streptomycine ont été ajoutés. Un volume de $100 \mu$, prélevé dans l'homogénat, a ensuite été étalé sur le milieu. A partir du troisième jour après l'étalement, les colonies de champignons ayant poussé ont été dénombrées, isolées et repiquées sur le même milieu dans d'autres boîtes de Pétri en vue de leur purification.

L'identification des champignons a été effectuée en s'appuyant sur leurs caractères morphoculturaux et microscopiques (Camara, 2011). Il s'est agi d'observer d'une part, le mycélium sur milieu de culture (coloration, aspect, allure de la croissance...) et d'autre part, les filaments, les conidiophores et les conidies grâce à un montage entre lame et lamelle d'un fragment de chaque champignon. L'identification des espèces fongiques a été faite à l'aide de la clé de détermination de Barnett et Barry (1972).

Les fréquences d'isolement des divers champignons ont été par ailleurs calculées en faisant le rapport du nombre de colonies d'un champignon donné sur le nombre total de colonies isolées. 


\section{Résultats}

\section{Types d'insectes infestant les fèves}

Les insectes rencontrés dans les échantillons sont des Ephestia cautella, appelés communément «mites ». Ils sont présents soit sous forme de larves à l'intérieur des fèves, soit à l'extérieur au stade adulte (figure 3). A l'âge adulte, ils laissent des traces de leur présence antérieure (parties des cotylédons rongés, détritus et toiles) dans les fèves.
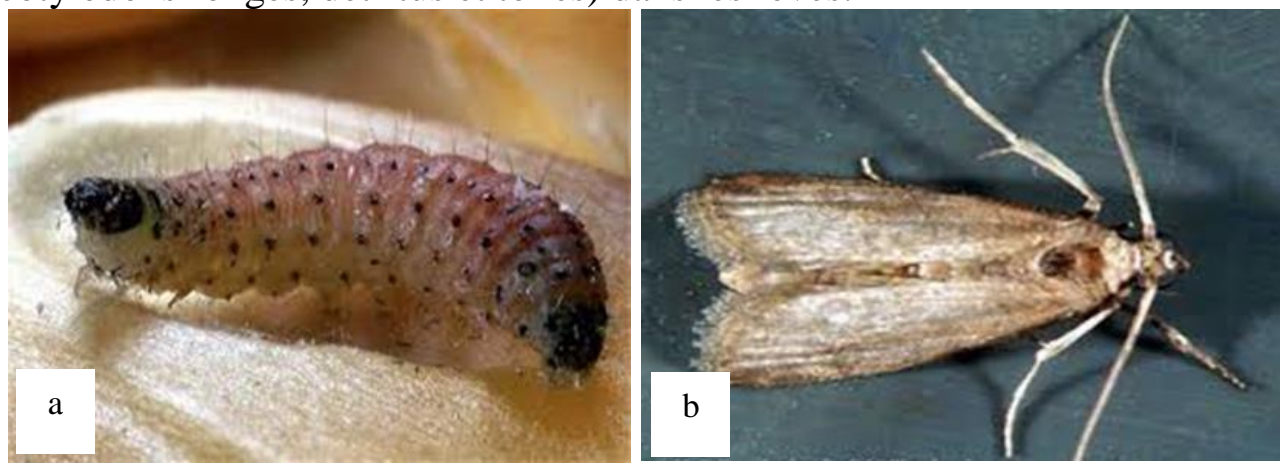

Figure 3 : Différents stades d'évolution d'Ephestia cautella: a : Stade larvaire $(\mathrm{G} \times 5)$ b : Stade adulte $(\mathrm{Gx} 4)$

\section{Types de moisissures contenues dans les fèves}

Les champignons retrouvés dans les fèves ont été identifiés à partir de leurs caractéristiques morphoculturales et microscopiques. Neuf espèces ont été retrouvées dans les fèves de cacao provenant des trois localités de production. Il s'agit de :

Aspergillus sp. 1 : Il présente sur milieu de culture PDA, un mycélium moyennement ras, à croissance régulière. Ce mycélium, blanc en début de croissance, devient noirâtre et granulé à maturité avec absence de coloration au verso de la boîte de Pétri. Au niveau microscopique, ce champignon présente des filaments mycéliens cloisonnés et ramifiés, d'où partent des conidiophores très longs $(1,5 \mathrm{à} 3 \mathrm{~mm})$ terminés par des têtes aspergillaires bisseriées (métules + phialides). Les conidies de forme globuleuse sont disposées en chaîne sur les phialides (figure 4).

Aspergillus sp. 2: Ce champignon présente, sur milieu PDA, un mycélium duveteux, blanchâtre à croissance rapide et nette. Le verso de la culture est jaunâtre. Au microscope, il a des filaments ramifiés et cloisonnés. Les conidiophores sont courts et portent des têtes aspergillaires sphériques ou ovoïdes sur lesquelles sont directement rattachées des conidies de forme également ovoïde (figure 5).

Aspergillus sp. 3 : Sur milieu de culture PDA, cet Aspergillus donne un aspect poudreux. Son mycélium moyennement ras est à croissance régulière et de couleur grisâtre. Son aspect microscopique est caractérisé par des filaments non ramifiés et cloisonnés. Les conidiophores sont également 
cloisonnés et portent des têtes aspergillaires bissériées (composées de phialides et de métules) sur lesquelles sont placées des spores en chaîne (figure $6)$.

Aspergillus sp. 4 : Le mycélium de cet Aspergillus, est moyennement ras, à croissance irrégulière, à aspect poudreux et de couleur gris-noirâtre. L'observation microscopique de ce champignon montre des filaments rectilignes, non cloisonnés, portant de longs conidiophores à têtes aspergillaires rondes sur lesquelles sont directement insérées des conidies de forme ovoïde (figure 7).

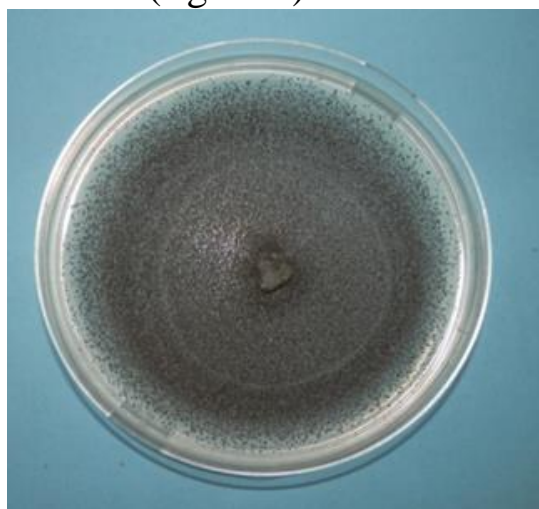

a : Aspect morphocultural $(\mathrm{G} \times 0,5)$

Figure 4 : Morphologie d'Aspergillus sp.1 sur milieu PDA

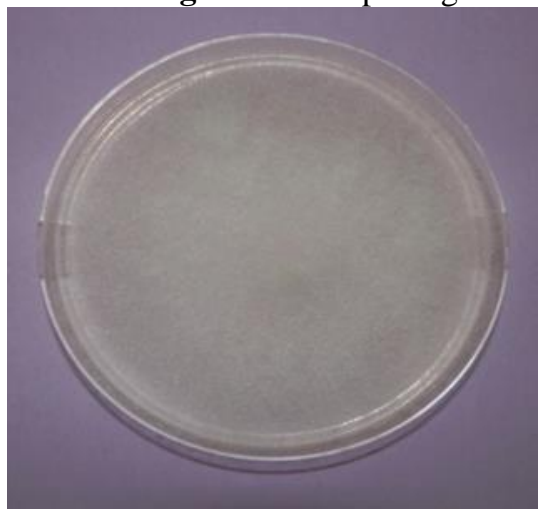

$\mathrm{a}:$ Aspect morphocultural $(\mathrm{G} \times \mathrm{0}$ 0,5) $\mathrm{b}$ : Aspect microscopique (G x 40)

Figure 5 : Morphologie d'Aspergillus sp. 2 sur milieu PDA

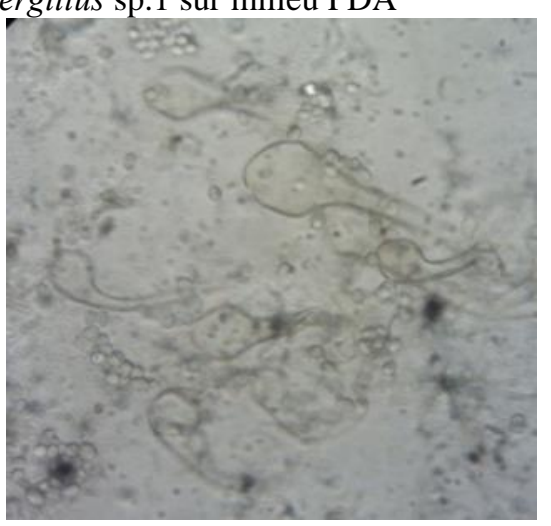




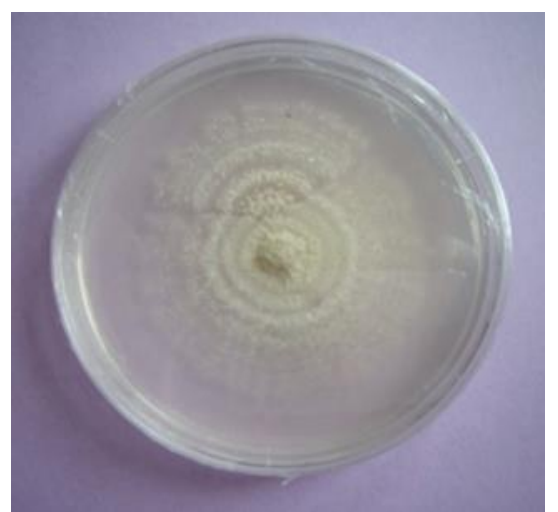

a : Aspect morphocultural $(\mathrm{G} \times 0,5)$

Figure 6: Morphologie d'Aspergillus sp.3 sur milieu PDA

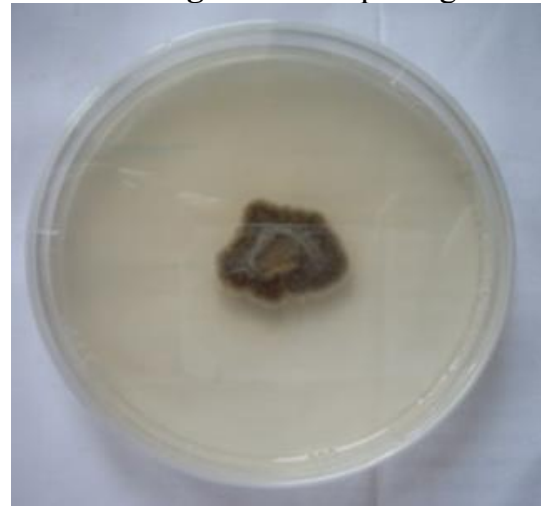

a : Aspect morphocultural $(\mathrm{G} \times 0,5)$

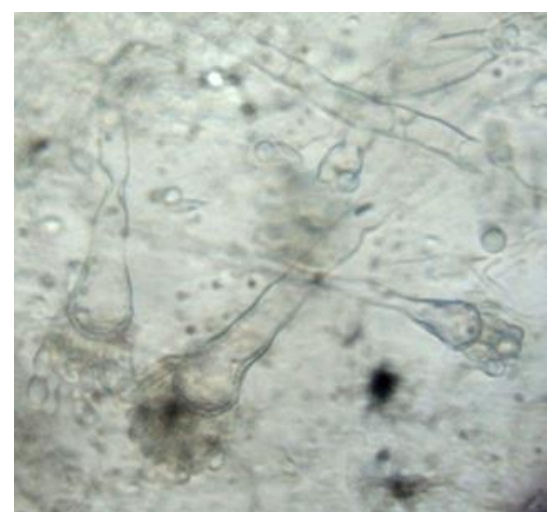

$\mathrm{b}$ : Aspect microscopique ( $\mathrm{G}$ x 40)

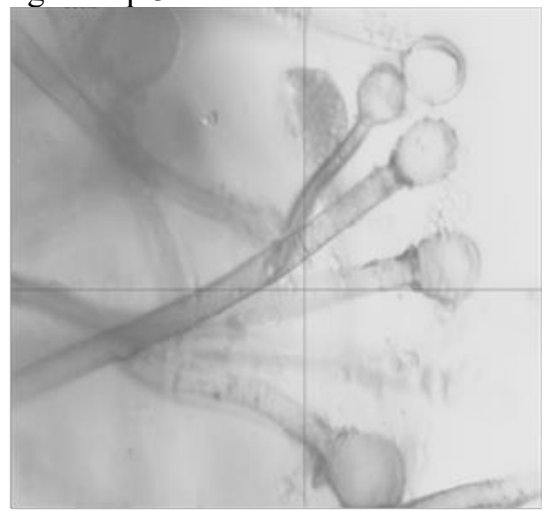

b : Aspect microscopique ( $\mathrm{G}$ x 40)

Figure 7 : Morphologie d'Aspergillus sp.4 sur milieu PDA

Aspergillus sp. 5: Le mycélium de ce champignon a un aspect granuleux, de couleur verte donnant un verso violet sur milieu PDA. La croissance du mycélium est régulière avec un front de croissance blanc. Au microscope, ce champignon présente des filaments fins, ramifiés et cloisonnés. Les conidiophores sont terminés par des têtes aspergillaires sphériques portant des spores qui y sont rattachées par des phialides (figure 8).

Fusarium sp. : Le faciès de ce champignon présente un mycélium aérien, cotonneux et jaunâtre. Ses filaments cloisonnés et ramifiés portent des microconidies et des macroconidies arquées et cloisonnées en 2 à 5 cellules. L'une des extrémités de ces conidies est pointue et l'autre, arrondie. (figure 9).

Streptomyces sp.: Le mycélium de Streptomyces sp. est ras, de couleur beige et à croissance régulière sur milieu PDA. Ses filaments ramifiés et cloisonnés portent des spores ovoïdes disposées en chaîne (figure 10).

Curvularia sp. : Le mycélium de Curvularia sp. est cotonneux, aérien de couleur noirâtre donnant un verso noir lors de sa culture sur milieu PDA. Les filaments mycéliens sont cloisonnés et portent des conidiophores terminés 
par des conidies sectionnées en 2 à 5 cellules dont une centrale est élargie (figure 11).

Penicillium sp. : Ce champignon a un mycélium moyennement aérien de couleur verdâtre à aspect poudreux donnant un verso de couleur jaunâtre sur milieu PDA. Les filaments, cloisonnés, portent des conidiophores terminés par des phialides en forme de pinceau, sur lesquelles se trouvent des conidies en chaîne (figure 12).
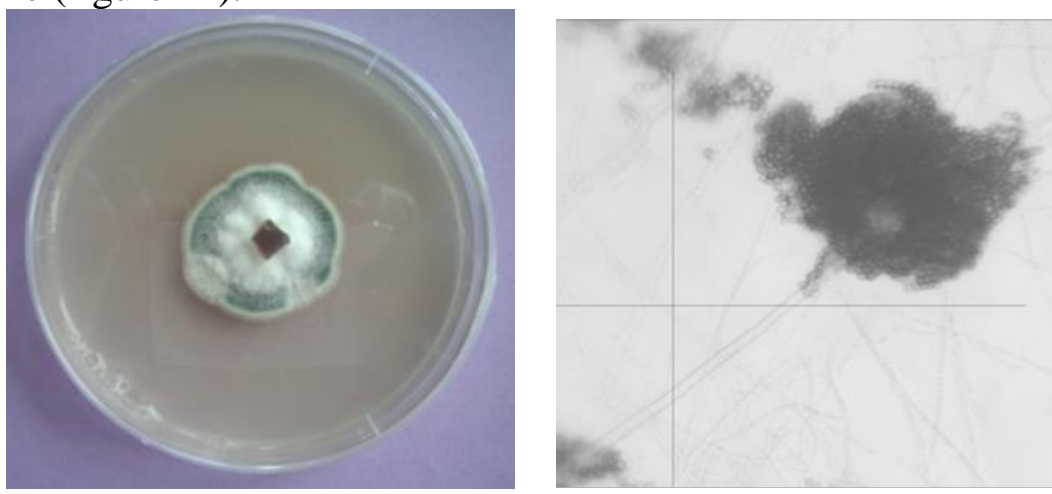

$\mathrm{a}:$ Aspect morphocultural (G x 0,5)

$\mathrm{b}$ : Aspect microscopique ( $\mathrm{G} \times 40)$

Figure 8 : Morphologie d'Aspergillus sp.5 sur milieu PDA

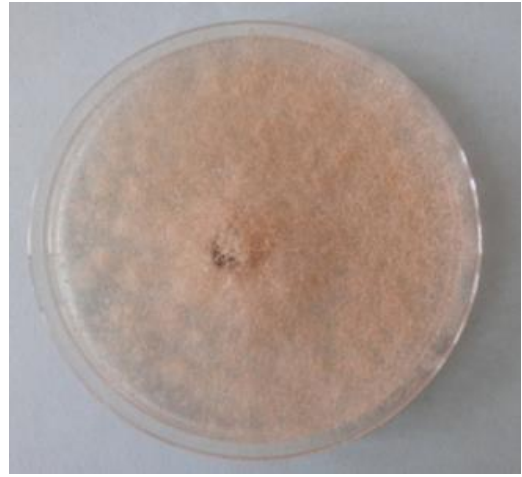

a : Aspect morphocultural $(\mathrm{G} \times 0,5)$

b : Aspect microscopique ( $\mathrm{G} \times 40)$

Figure 9 : Morphologie de Fusarium sp. sur milieu PDA
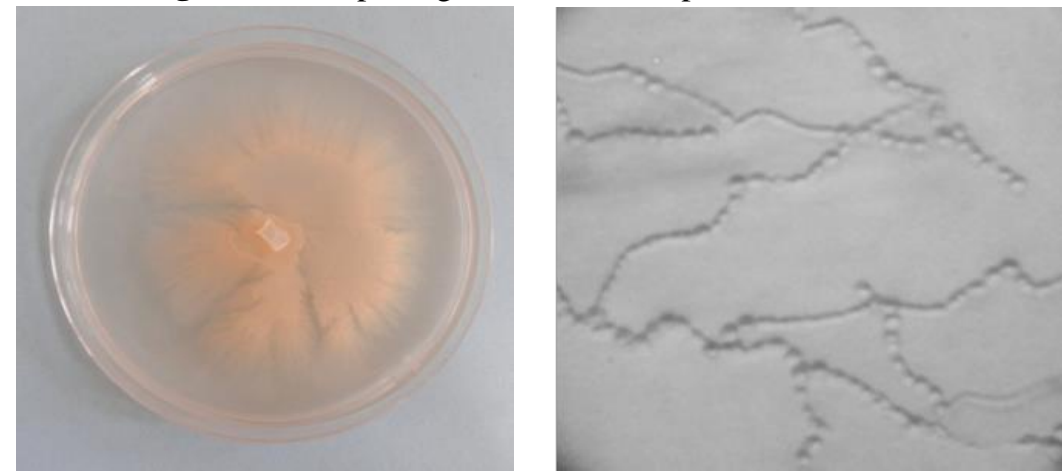

$\mathrm{a}$ : Aspect morphocultural $(\mathrm{G} \times 0,5)$ b : Aspect microscopique $(\mathrm{G} \times \mathrm{40})$

Figure 10 : Morphologie de Streptomyces sp. sur milieu PDA 

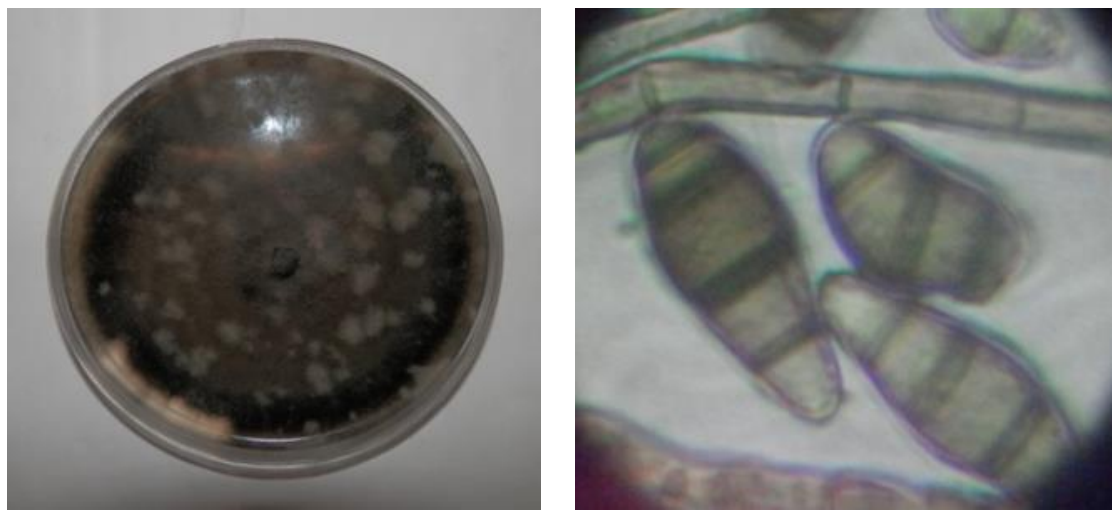

$\mathrm{a}$ : Aspect morphocultural (G x 0,5) b : Aspect microscopique (G x 40) Figure 11 : Morphologie de Curvularia sp. sur milieu PDA
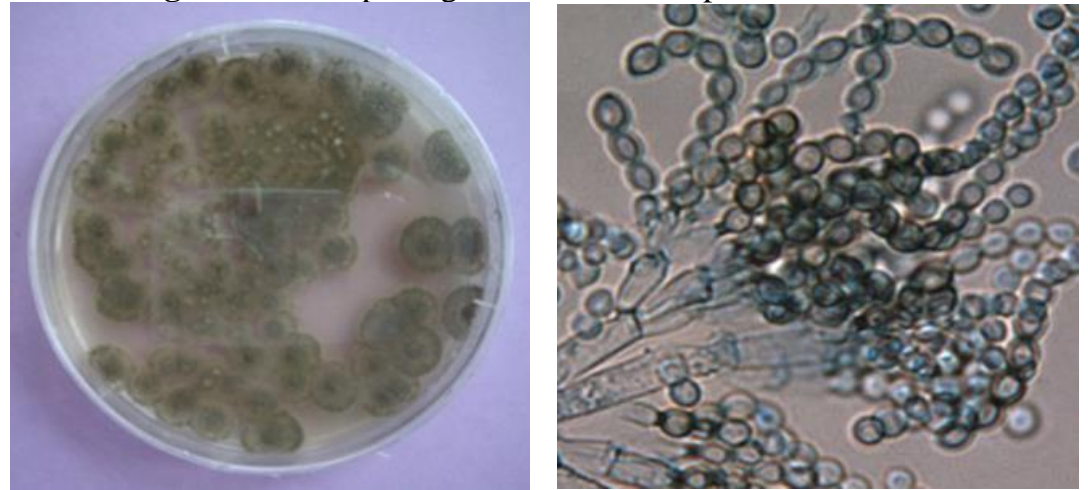

$\mathrm{a}$ : Aspect morphocultural (G x 0,5) b : Aspect microscopique (G x 40)

Figure 12 : Morphologie de Penicillium sp. sur milieu PDA

\section{Fréquence de contamination des fèves par les champignons}

La figure 13 présente les espèces de champignons isolées dans les principales localités de production. A Abengourou, cinq (5) champignons (Fusarium sp., Streptomyces sp., Curvularia sp., Aspergillus sp.1 et Aspergillus sp.2) ont été rencontrés. Parmi eux, les champignons du genre Aspergillus (Aspergillus sp.1 et Aspergillius sp.2, isolés à des fréquences respectives de $71,25 \%$ et $22,71 \%$ ) ont dominé la population fongique dans les fèves. A Oumé, cinq (5) champignons ont également été isolés. Il s'agit de Aspergillus sp.1, Aspergillus sp.2, Aspergillus sp.3, Aspergillus sp.4 et Penicillium sp.. Dans cette localité, les champignons prédominants ont été Aspergillus sp.1 (73,96 \%) et Penicillium sp (11,32 \%). A Soubré, quatre (4) champignons contaminant les fèves ont été trouvés, dont deux du genre Aspergillus (Aspergillus sp.1 et Aspergillus sp.5), un du genre Streptomyces et un autre du genre Penicillium. Les champignons majoritairement isolés ont été, Aspergillus sp.5 (75,61\%) et Streptomyces sp. (18,97\%).

D'une manière générale, 9 espèces de champignons ont été retrouvées dans les fèves de cacao collectées dans l'ensemble des trois localités de 
production choisies (figure 14). Parmi ces champignons, Aspergillus sp.1 et Aspergillus sp.5 ont été très fréquemment rencontrés (respectivement 49,33 et 25,30 \%). Viennent ensuite Aspergillus sp.2 (9,43\%), Streptomyces sp. (7,89 $\%)$, Penicillium sp. (4,46\%), Aspergillus sp.3 (1,88 \%) et Aspergillus sp.4 $(1,01 \%)$. Fusarium sp. et Curvularia sp. ont été isolés à de très faibles fréquences (moins de $1 \%$ ).

Par ailleurs, la plupart des champignons rencontrés dans les fèves de cacao collectées appartiennent au genre Aspergillus. En effet, ce genre représente $86,52 \%$ des champignons isolés dans l'ensemble des localités de production (Figure 15).

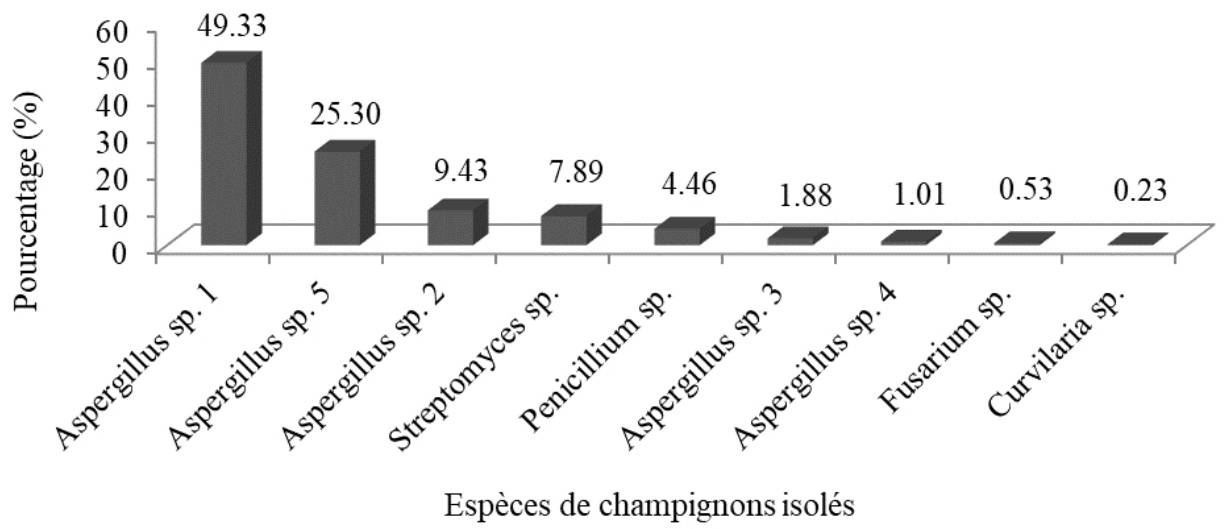

Figure 13 : Fréquences de contamination des fèves de cacao par les espèces de champignons sur l'ensemble des localités étudiées

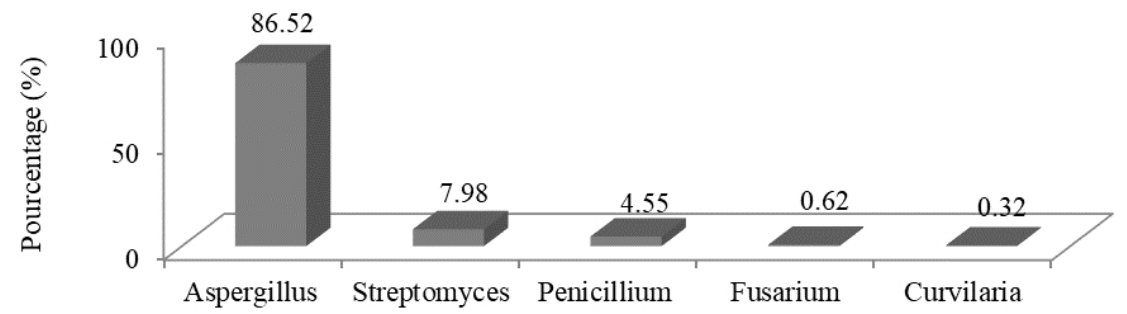

Genres de champignons isolés

Figure 14 : Fréquences de contamination des fèves de cacao par les différents genres de champignons sur l'ensemble des localités étudiées 

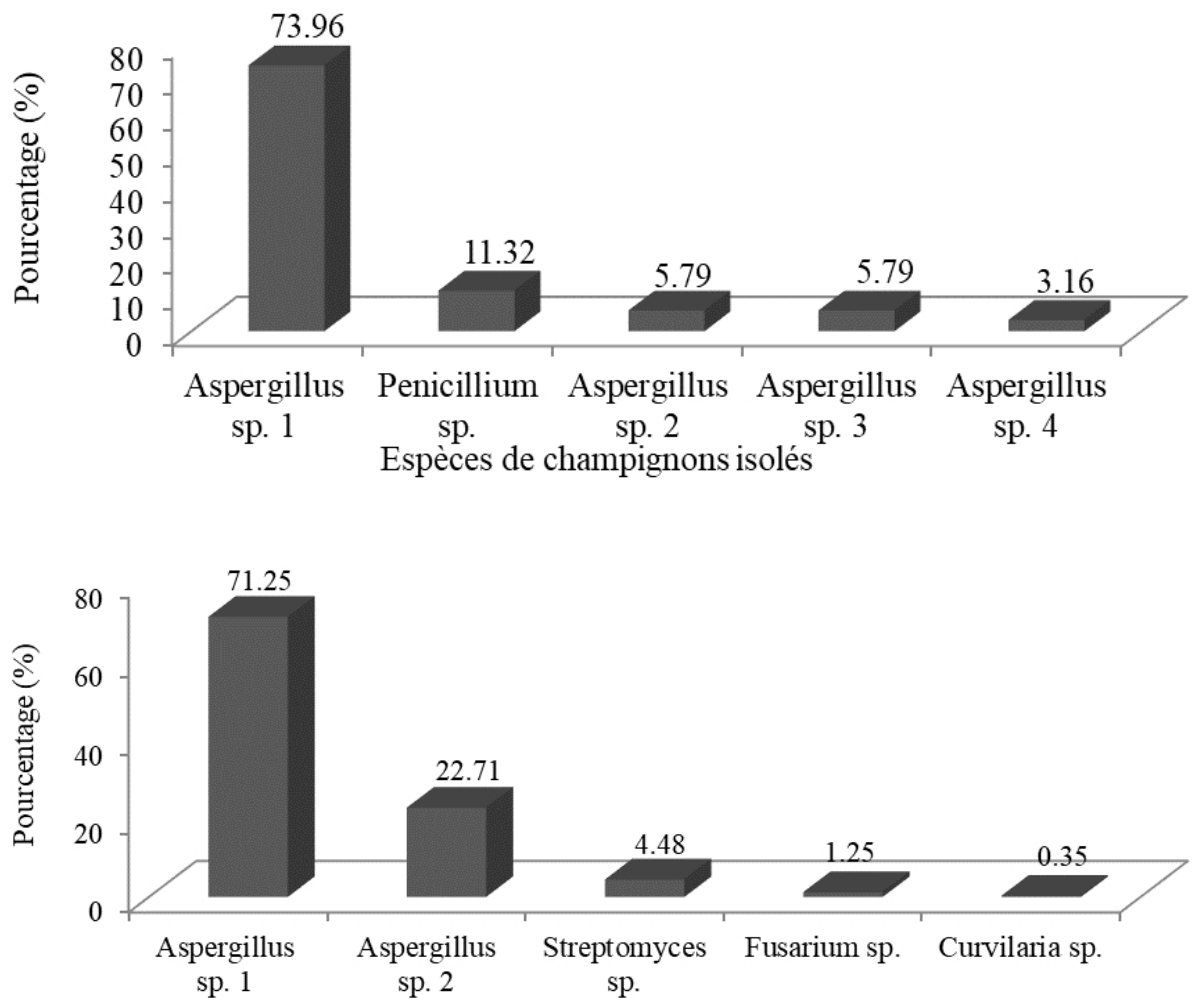

Espèces de champignons isolés

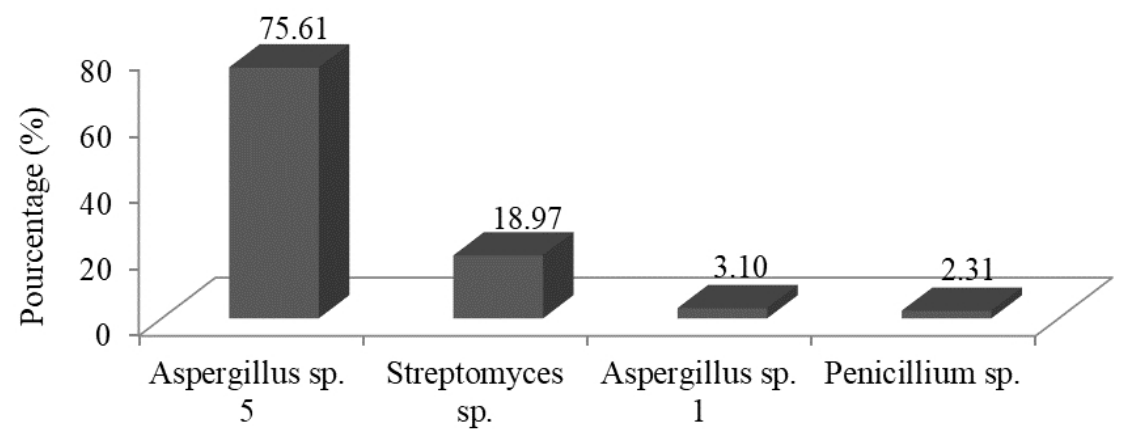

Espèces de champignons isolés

Figure 15 : Fréquences d'apparition des espèces de champignons en fonction des localités

\section{Discussion}

Les fèves de cacao collectées dans les principales zones de production en Côte d'Ivoire ont été infestées par des insectes et des champignons.

Ephestia cautella est l'insecte qui a été retrouvé dans les fèves collectées. Cet insecte a déjà été cité parmi ceux qui attaquent les denrées 
alimentaires, surtout lors de leur conservation (Ndiaye, 1999). Une forte présence d'insectes dans le cacao est un défaut commercial car leur taux est pris en compte dans la détermination du grade du cacao (BCC, 2006). Les taux d'infestations qui étaient pratiquement nuls dans l'ensemble des échantillons avant le test de conservation, sont passés à 26,67\% à Abengourou, 30,67 \% à Oumé et 25,00\% à Soubré. Ces derniers montrent une apparition ultérieure d'insectes dans les échantillons après leur collecte, précisément lors de l'entreposage des fèves. Ces contaminations seraient survenues durant le processus de préparation des échantillons. En effet, avec le dispositif qui exclut toute possibilité de contamination d'origine extérieure des fèves pendant le stockage, il est évident que l'augmentation de ces taux d'infestation est due à une apparition d'insectes suite à une éclosion de leurs œufs qui se trouvaient préalablement sur ces fèves lors de l'échantillonnage. Les problèmes d'infestation rencontrés dans les stocks de cacao des magasins et entrepôts sont certes dus à un mauvais entretien de ces lieux (Barel, 2013), mais aussi à une infestation initiale des fèves dans les zones de production. Cette infestation initiale proviendrait, soit des matériels utilisés durant les traitements technologiques (séchoirs et sacs) qui contenaient déjà des œufs d'insectes, soit de la ponte d'œufs par les insectes durant le bref délai qui sépare la fin du séchage et la vente de la marchandise par le producteur (Braudeau, 1969). Dans tous les cas, les contaminations subséquentes diminuent la valeur marchande des produits en les faisant passer d'un grade donné à un autre inférieur, surtout quand le préstockage est prolongé. Même lorsque la vente est immédiate, ces contaminations engendrent à long terme dans les entrepôts, des proliférations d'insectes nécessitant des désinsectisations à coûts souvent élevés.

L'isolement et l'identification des moisissures infestant les fèves de cacao ont révélé que le genre Aspergillus est le plus rencontré à la surface et à l'intérieur des fèves. En effet, ce genre a représenté $86,30 \%$ des genres de moisissures isolés. Ces résultats confirment ceux de Dongo et al. (2006) et Guéhi et al. (2007) qui ont montré la prédominance du genre Aspergillus dans la flore fongique contaminant les fèves de cacao. Les taux élevés de fèves moisies constitue un défaut commercial. (BCC, 2006). De plus, ces champignons, pour la plupart, sont susceptibles de produire des toxines redoutées par les consommateurs de produits à base de cacao (Mounjouenpou, 2009 ; Kouadio 2012).

\section{Conclusion}

Les fèves de cacao produites dans les principales zones de production en Côte d'Ivoire sont susceptibles d'être infestées par des champignons et des insectes de la fin de la production à la commercialisation ou à la transformation. Les insectes trouvés dans les fèves de cacao sont du genre 
Ephestia catella. Leurs œufs contaminent les fèves pendant le processus postrécolte du cacao marchand et éclosent les moments qui suivent la vente. Quant aux champignons, ils sont dominés par le genre Aspergillus reconnus producteurs de toxines. Les spores se retrouvent déjà sur les fèves et prolifèrent ultérieurement, surtout quand les conditions sont favorables au développent des moisissures. Le bon séchage des fèves, l'utilisation d'emballages propres non contaminés et le respect des bonnes conditions de stockage devraient contribuer à réduire les niveaux d'infestation des fèves marchandes.

\section{References:}

1. Aneani F. et Takrama JF., 2006. Les pratiques qui influencent la qualité du cacao au Ghana: la perception des cacaoculteurs. 15 ème conférence internationale sur la recherche cacaoyère, San-José, Costa Rica, 9-14 octobre 2006.

2. Barel M., 2013. Qualité du cacao : l'impact de traitement post-récolte. Collection Savoir-faire, Quae ed, 94 p.

3. Barnett H. L. et Barry B. H., 1972. Illustrated Genera of Imperfect Fungi. Burgess Publishing Incorporated, 241 p.

4. Bastide P., Fourny G., Durand N., Petithuguenin P., Guyot B., Gilmour M. et Lindblom M., 2010. Identification des sources d'ochratoxine A durant la phase post-récolte du cacao: influence de la qualité de la récolte et des facteurs climatiques. In : Proceed 15th International Cocoa Research Conference, 2006-10-09/2006-10-14, San José, Costa Rica, Cocoa Producers' Alliance, pp1171-1178.

5. BCC (Bourse du Café et du Cacao), 2006. Système d'Information des marchés. Note de conjoncture n 16 , février/Juillet 2006 - du 15 Août 2006.

6. Braudeau J., 1969. Le cacaoyer ; Maisonneuve et Larose (eds), Paris, 302 p.

7. Camara B., 2011. Caractérisation des parasites fongiques foliaires et telluriques en Côte d'ivoire chez les bananiers (Musa sp) et recherche de méthodes de lutte. Thèse de Doctorat de L'Université de Cocody Abidjan, UFR Biosciences, Abidjan, 237 p.

8. Cros E. et Jeanjean N., 1995. Qualité du cacao. Influence de la fermentation et du séchage. Plantation, Recherche et Développement, 2(3): 21-27.

9. Dongo I. N., Bandyopadhyay R. et Jayeola C. O., 2006. Etat de la contamination des fèves de cacao liée à la présence de mycotoxines au Nigéria et ses implications pour le commerce. $15^{\text {ème }}$ conférence internationale sur la recherche cacaoyère, San-José, Costa Rica, 9-14 octobre 2006. 
10. FAO (Food and Agriculture Organization), 1999. Preventing mycotoxin contamination. In: Food nutrition and agriculture $\mathrm{N}^{\circ} 23$. Rome: Food Nutrition Division, FAO.

11. IARC (International Agency for Research on Cancer), 1993. Ochratoxin A. Monograph on the evolution of carcinogenic risk to humans. Lyon, France, IARC Work Group,World Health Organisation, pp250-277.

12. ICCO (Organisation internationale du cacao), 2005. Bulletin trimestriel de statistiques du cacao Vol. XXX n ${ }^{\circ} 4$.

13. Jarrige F. et Ruf F., 2000. Comprendre la crise du cacao. Café Cacao Thé, vol. XXXIV (3) : 213-222.

14. Kouadio J. H., 2012. Ochratoxine A en Côte d'Ivoire : moisissures ochratoxinogènes, exposition humaine et détoxification des aliments. Revue Ivoirienne des Sciences Technologiques,. 20 (2012) 87-103.

15. Kouakou B.J., IRIE B. Z. DICK E., Nemlin N.Bomisso E. L., 2013. Caractérisation des techniques de séchage du cacao dans les principales zones de production en Côte d'Ivoire et détermination de leur influence sur la qualité des fèves commercialisées. Journal of Applied Biosciences 64 : 4797-4812.

16. Lery F., 1974. Le cacao. Que sais-je ? Presse Universitaire de France, Paris 46p.

17. Mounjouenpou P., Gueule D., Fontana-Tachon, A., Guyot B. et Guiraud J. P., 2009. Qualité du cacao camerounais: Facteurs influençant la présence et l'activité des moisissures productrices d'ochratoxine A. In : Transformation, conservation et qualité des aliments : nouvelle approche de lutte contre la pauvreté pp141-148.

18. Ndiaye D. S. B., 1999. Manuel de stockage et de conservation des céréales et des oléagineux, $61 \mathrm{p}$.

19. Stanek V. J., 1979. Encyclopédie illustrée des insectes. $6^{\mathrm{e}}$ éd. Gründ, Prague, $548 \mathrm{p}$. 